\title{
DESIGUALDAD DE GÉNERO, INCLUSIÓN LABORAL Y RIESGOS PSICOSOCIALES: EVIDENCIAS EN TRABAJADORAS CON DISCAPACIDAD INTELECTUAL
}

\section{Noelia Flores \\ nrobaina@usal.es}

Universidad de Salmanca

Paula Martinelli

martinellipaula@hotmail.com

Fundación Síndrome de Down Madrid
Cristina Jenaro

crisje@usal.es

Universidad de Salmanca

\author{
Vanessa Vega
}

vanejuay@gmail.com

Universidad Católica de Valparaíso - Chile

Recibido: 28-02-2014

Aceptado: 06-03-2014

\section{Resumen}

El empleo es un factor clave para garantizar la igualdad de oportunidades. Las mujeres con discapacidad intelectual se encuentran en una situación de doble desventaja que dificulta su inclusión laboral. El objetivo de este trabajo consiste conocer las condiciones en las que se desarrolla el empleo así como los factores de riesgo psicosocial en trabajadoras con discapacidad intelectual. Se presentan los resultados obtenidos tras la evaluación de 64 mujeres pertenecientes a empleo con apoyo (ECA). Se constata que las mujeres experimentan riesgos psicosociales y que el estrés y burnout afectan a su salud laboral. También se identifican factores que potencian la satisfacción en estas trabajadoras. Finalmente se proponen estrategias para la prevención.

Palabras Clave: Desigualdad de género, inclusión laboral, riesgos psicosociales, discapacidad intelectual, empleo con apoyo, satisfacción laboral.

\begin{abstract}
Employment is the key to ensure equal opportunities. Women with intellectual disabilities are at a double disadvantage situation, what hinders their labor inclusion. The objective of this work is to identify employment conditions and psychosocial risk factors in female workers with intellectual disabilities. Results obtained after assessing 64 female supported employees (ECA) are presented. Results showed that women experience psychosocial risks and that the stress and burnout affect their occupational health. The factors that enhance job satisfaction are also identified. Finally, prevention strategies are proposed.
\end{abstract}

Keywords: Gender Inequality, labor inclusion, psychosocial risks, intellectual disabilities, support employment, work satisfaction. 


\section{Introducción}

El empleo es un factor esencial para garantizar la igualdad de oportunidades de todas las personas y contribuye, de manera decisiva, a la plena participación de la ciudadanía en la vida económica, social y cultural (Nuño y Torres, 2012). Se trata, por tanto, de la mejor herramienta de inclusión social que confiere una organización a la vida de las personas y contribuye, a su vez, al bienestar económico y social de la comunidad (Super, 1980). De hecho, parece existir acuerdo respecto a los beneficios o funciones positivas (p.e. auto-realización, fuente de identidad, estatus económico y social, contactos interpersonales, etc.) que aporta a la vida de las personas (Salanova, Peiró y Prieto, 1999). Ahora bien, la realidad laboral se halla muy diversificada y mientras que en algunos trabajos la mayor parte de las funciones están garantizadas, en otros, las condiciones son tales que no permiten lograr dichos aspectos, repercutiendo negativamente en la salud de la persona y en la propia organización (Kelloway y Day, 2005; Salanova, Gracia y Peiró, 1996). Por otro lado, también se dan numerosas situaciones de discriminación en el mercado de trabajo que afectan, principalmente, a las personas vulnerables o en situación de vulnerabilidad social (CERMI, 2012).

En el caso de las mujeres su lucha y esfuerzo por incorporarse al mercado laboral en condiciones de igualdad ha provocado que se produzca, en las últimas décadas, un creciente interés político y social con el objetivo de intervenir para garantizar la igualdad entre hombres y mujeres e intentar eliminar la discriminación existente por razón de género (Marcos, 2009; Nuño y Torres, 2012; Silva, 2008). En relación al colectivo de personas con discapacidad, es posible evidenciar que, aún hoy, y pese a los avances principalmente en cuanto a instrumentos jurídicos para promover la igualdad de oportunidades y luchar contra la discriminación, alcanzados en los últimos años, subsisten las limitaciones en el uso y disfrute de los derechos sociales y económicos (CERMI, 2012); algo que repercute de forma directa en el mercado laboral, y sitúa al trabajador con discapacidad en una clara situación de desventaja respecto a sus iguales sin dicha condición (Pallisera y Rius, 2007).

De manera particular, las mujeres con discapacidad se enfrentan a un reto aún mayor dado que no se encuentran en la misma situación que el resto de mujeres, ni tampoco están en las mismas condiciones que los hombres con discapacidad, por lo que se 
suele hablar de una "doble discriminación"-por género y discapacidad- (Conde, Portillo y Shum, 2003). En este sentido la discapacidad y el género interactúan situando a las mujeres con discapacidad en una posición desigual en el mercado de trabajo. Dicha situación se refleja en los datos ofrecidos por la última Encuesta de Población Activa y de la Base Estatal de Personas con Discapacidad en el año 2012 (INE, 2013), donde encontramos que en 2012 había un total de 1.450 .800 personas con certificado de discapacidad en edad de trabajar (de 16 a 64 años), lo que supone un 4,8\% de la población total en edad laboral. Así mismo, el 36,6\% de las personas con discapacidad legalmente reconocida eran activos en 2012. Dicha tasa de actividad era de 40 puntos inferior a la de la población sin discapacidad (77\%). Por otro lado, la tasa de empleo de las personas con certificado de discapacidad era de un $24,5 \%$ frente al $57,8 \%$ de sus iguales sin discapacidad. Atendiendo a los datos de las personas con discapacidad según el género, encontramos que en la población activa con discapacidad se observa un mayor porcentaje de hombres $(58,2 \%)$ frente al de mujeres $(41,8 \%)$. La tasa de empleo de los hombres con certificado de discapacidad fue de un $26,2 \%$ frente al $22,5 \%$ de las mujeres. En lo que respecta a los datos de las mujeres, se constata también que la tasa de actividad en las mujeres con discapacidad fue de un $33,5 \%$ frente al $70,4 \%$ de sus homónimas sin discapacidad. Por otro lado, la tasa de empleo de las mujeres con discapacidad se situó en el $22,5 \%$ frente al $52,6 \%$ de las mujeres sin discapacidad. Por tanto, al igual que sucede en la población general, las mujeres con certificado de discapacidad tienen una menor presencia activa en el mercado laboral.

Estos datos ponen de manifiesto la situación de exclusión que se sigue produciendo en el mercado laboral para las personas con discapacidad en general y para las mujeres en particular. Se hace necesario, por tanto, poner en marcha medidas encaminadas a disminuir la brecha existente entre mujeres y hombres con discapacidad y el resto de la población, dado que existen numerosas evidencias que han puesto de manifiesto que las personas con discapacidad pueden desempeñar con éxito un trabajo y contribuir a la comunidad (Flores, Jenaro, Orgaz y Martín, 2011; Mank, Cioffi y Yovanoff, 1997; Winer, 2000). Por otro lado, también se ha evidenciado que el trabajo mejora la autoestima y la valoración social de estas personas (Alomar, 2004; Jenaro, 2004; Pallisera y Rius, 2007). Además, numerosos estudios han confirmado que el empleo es un factor que mejora la calidad de 
vida de este colectivo (Eggleton, Robertson, Ryan y Kober, 1999; Jenaro y Bagnato, 2006).

Por tanto, la inclusión laboral de las personas con discapacidad ha de cumplir una triple finalidad: objetiva, integradora y socioeconómica (Berg, 1981), dado que posibilita: (a) el ejercicio del derecho al trabajo, (b) el aprendizaje social y la mejora en la calidad de vida y, (c) el desarrollo profesional y económico. La Convención sobre los Derechos de las Personas con Discapacidad también reconoce en su artículo 27 el derecho de las personas con discapacidad a trabajar en igualdad de condiciones y a ganarse la vida mediante un trabajo libremente elegido o aceptado en un mercado y un entorno laboral que sea abierto, inclusivo y accesible a las personas con discapacidad (ONU, 2006:18). Para ello, los Estados Partes deberán salvaguardar y promover el ejercicio del derecho al trabajo, incluso para las personas que adquieran una discapacidad durante el empleo, adoptando las medidas pertinentes para prohibir la discriminación por motivos de discapacidad en el empleo. Dicho artículo hace además referencia a la obligación de proteger los derechos de las personas con discapacidad en el entorno laboral en igualdad de condiciones con las demás personas (CERMI, 2013).

$\mathrm{Y}$ todo ello porque, entre otras cosas, tener un empleo per se no es suficiente para garantizar la satisfacción de un trabajador con discapacidad (Farris y Stancliffe, 2001; Jenaro et al., 2002). Será necesario asegurar un adecuado equilibrio entre la normalización o la similitud de las condiciones de trabajo con las existentes para los trabajadores en general, y los apoyos, es decir, entre las exigencias del trabajo y los recursos disponibles. Por tanto, la satisfacción laboral de un trabajador con discapacidad es una condición que va más allá de la mera obtención de un empleo o del tipo de entorno donde se encuentre trabajando. Dicha satisfacción está determinada también por el ambiente de trabajo experimentado por el trabajador, por la flexibilidad de dicho ambiente y por el adecuado equilibrio entre las características de la tarea y las habilidades del trabajador (Flores, 2008; Jenaro et al., 2002).

Una de las principales responsabilidades de los poderes públicos es la de velar por la salud y la seguridad e higiene en el trabajo (Peiró y Bravo, 1999). Dichas consideraciones aparecen en el Artículo 136 del Tratado de Roma que establece como objetivo prioritario para la Unión Europea y sus Estados miembros promover la mejora del medio de trabajo con el fin de proteger la seguridad, la salud y las condiciones laborales de 
los trabajadores (Parent-Thirion, Fernández, Hurley y Vermeylen, 2007). En nuestro país, este acuerdo se concreta en la Ley de Prevención de Riesgos Laborales (LPRL, Ley 31/1995 de 8 de noviembre) que tiene como objeto promover la seguridad y la salud de los trabajadores mediante la aplicación de medidas y el desarrollo de las actividades necesarias para la prevención de riesgos en el trabajo. Partiendo de esta consideración, y desde un punto de vista psicosocial, los factores de riesgo se definen como aquellas condiciones que están presentes en una situación laboral, directamente relacionadas con la organización, el contenido del trabajo y la realización de la tarea, y que pueden afectar tanto al desarrollo del trabajo como a la salud (física, psíquica o social) del trabajador (Sauter, Hurrell, Murphy y Levi, 2001). Los factores de riesgo susceptibles de encontrarse en el medio ambiente de trabajo pueden ser numerosos y de diferente naturaleza. A éstos se les suele denominar "estresores" puesto que su exposición puede repercutir tanto en la salud física como psicológica del trabajador. Además, si la situación de estrés persiste en el tiempo, puede dar lugar al denominado síndrome de burnout o síndrome de quemarse por el trabajo.

En el caso de los trabajadores con discapacidad, el artículo 25.1 de la LPRL establece que los empresarios deben garantizar la protección de aquellos trabajadores especialmente sensibles a determinados riesgos derivados del trabajo, entre los que se encuentran las personas que tengan reconocida su situación de discapacidad física, psíquica y sensorial. Consecuentemente, el análisis de los principales riesgos psicosociales a los que pueden estar expuestos estos trabajadores debe ser también un objetivo para todas aquellas organizaciones preocupadas por la mejora de la salud y calidad de vida laboral de sus empleados. En Europa, por ejemplo, la Agencia Europea para la Seguridad y Salud en el Trabajo (2004) señala que las personas con discapacidad deben recibir un tratamiento equitativo en el trabajo, particularmente en lo que se refiere a la salud y seguridad laboral. Pese a ello, son escasos los estudios realizados en el colectivo de trabajadores con discapacidad. No obstante, algunas investigaciones a en el ámbito anglosajón concluyen que estos trabajadores también se exponen a situaciones de riesgo (i.e. estrés) en sus lugares de trabajo (Lunsky y Bramston, 2006; Nucci y Reiss, 1987) y se ven afectados por el mismo tipo de estresores que la población general (Bramston y Fogarty, 1995). De hecho, en una guía sobre el estrés publicada por la Comisión Europea sobre Seguridad y Salud en el trabajo (Levi y Levi, 2000) se constata que las personas con 
discapacidad intelectual constituyen un grupo de alto riesgo a padecer estrés en sus lugares de trabajo. Otros trabajos realizados en nuestro país también coinciden en señalar la existencia de demandas laborales y la relación de éstas con la calidad de vida laboral experimentada por el trabajador con discapacidad intelectual (Flores, Jenaro, Orgaz y Martín, 2011; Paredes, Fernández-Cid y Ruíz-Figueroa, 2012).

No obstante, son aún escasos los estudios sobre salud laboral y riesgos psicosociales en el colectivo de personas con discapacidad y mucho más aún aquéllos que hayan incorporado la perspectiva de género. En nuestro país, un estudio realizado Muriel (2011) aborda, mediante el análisis de las historias de vida, la situación laboral de las mujeres con discapacidad intelectual y enfermedad mental desde una perspectiva de género. Sin embargo, en dicho estudio no se alude a los riesgos laborales que éstas pueden experimentar. Por su parte, la Agencia Europea para la Seguridad y Salud en el Trabajo (2010) subraya que la diversidad rara vez ha sido contemplada en la evaluación de riesgos y que las herramientas de evaluación de riesgos específicos a los que se exponen, por ejemplo, las personas con discapacidad o las mujeres, entre otros colectivos, siguen siendo poco frecuentes. Por tanto, abordar esta realidad es de vital importancia para mejorar la inclusión laboral en condiciones de igualdad del colectivo de mujeres con discapacidad intelectual.

Partiendo de estas consideraciones, el presente trabajo pretende contribuir al conocimiento de los factores del entorno laboral que pueden suponer un obstáculo o bien potenciar la inclusión laboral de las mujeres con discapacidad intelectual desde una perspectiva de género. Concretamente, nos planteamos los siguientes objetivos: 1) Conocer los factores de riesgo psicosocial (demandas y falta de recursos laborales), el estrés laboral, el burnout y otras variables relacionadas con la salud laboral de las trabajadoras; 2) Determinar semejanzas y diferencias en demandas laborales, recursos, satisfacción y calidad de vida laboral en función del estrés percibido por las participantes; 3) Determinar semejanzas y diferencias en los factores de riesgo psicosocial en función de los niveles de burnout (agotamiento y cinismo) experimentados por las trabajadoras; y 4) Conocer otros factores potenciadores de la satisfacción en el trabajo (i.e. aspectos formativos, apoyos), a partir de la opinión de las propias trabajadoras con discapacidad intelectual. 


\section{Método}

\subsection{Participantes}

Las participantes en este estudio fueron una muestra de conveniencia formada por 64 mujeres trabajadoras con discapacidad intelectual que se encontraban trabajando en diferentes alternativas de empleo con apoyo (ECA). El Empleo con Apoyo es una modalidad de inserción laboral para personas con discapacidad que surge en nuestro país en 1989 y que está regulada por el Real Decreto 870/2007, de 2 de julio, como medida de fomento de empleo de personas con discapacidad en el mercado ordinario de trabajo. Se trata del conjunto de acciones de orientación y acompañamiento individualizado en el puesto de trabajo que tienen por objeto facilitar la adaptación social y laboral de trabajadores con discapacidad con especiales dificultades de inserción laboral, en empresas del mercado ordinario de trabajo y en condiciones similares al resto de los trabajadores que desempeñan puestos equivalentes.

Respecto al tipo de discapacidad, todas las participantes presentaban discapacidad intelectual como diagnóstico principal y sus niveles de discapacidad oscilaban entre límites y ligeros. Respecto a la edad de las participantes, predominaban aquellas con edades comprendidas entre los 22 a 30 años $(54,7 \%)$ seguidas quienes tenían entre 31 y 40 años $(42,2 \%)$. Tan sólo dos trabajadoras manifestaron tener más de 41 años (3,1\%). Así mismo, se observó un predominio de mujeres solteras (97\%), frente a quienes estaban casadas o conviviendo en pareja (3\%). La totalidad de las participantes manifestó no tener cargas familiares. En cuanto al tipo de trabajo realizado, éste se centraba fundamentalmente en auxiliar de oficinas y administrativo $(78,1 \%)$ frente a quienes trabajaban como reponedoras de almacén (11\%) o quienes trabajaban en imprentas o como ayudantes de ordenanzas y/o recepcionistas (10,9\%). La gran mayoría de las participantes, 52 (81,3\%), afirmaron tener un contrato indefinido, y tan sólo 12 (18,8\%) estaban contratadas de manera temporal. Respecto al tipo de jornada laboral, 52 mujeres trabajaban a media jornada $(81,3 \%)$ frente a $12(18,8 \%)$ que lo hacían a jornada completa. El trabajo lo desempeñaban mayoritariamente en jornada de mañana (97\%) frente a dos trabajadoras (3\%) que trabajan en turno de tarde. Respecto al tiempo de antigüedad en el puesto, un elevado porcentaje de las participantes $(59,4 \%)$ se caracterizó por llevar entre 1 y 5 años en su trabajo, seguidas de quienes llevaban entre 6 y 8 años (15,6\%). Tan sólo 8 trabajadoras (12,5\%) indicaron tener más de 11 años de antigüedad 
en el puesto. Así mismo, seis trabajadoras (9,4\%) manifestaron llevar menos de un año. Todas las mujeres se encontraban en activo en el momento del estudio y trabajaban en diferentes empresas de empleo ordinario ubicadas en la Comunidad de Madrid. Todas ellas accedieron a participar de manera voluntaria y ofrecieron su consentimiento informado.

\subsection{Instrumentos}

En la evaluación de los factores de riesgo psicosocial (demandas y falta de recursos laborales) se empleó el Cuestionario sobre el Contenido del Trabajo (traducido y adaptado de Karasek, 1985, por Jenaro y Flores, 2006). Este instrumento ha sido ampliamente utilizado en los contextos laborales y permite una evaluación válida y fiable de los aspectos más significativos que hacen referencia al contenido del trabajo. El cuestionario está compuesto por 34 items que se responden en una escala tipo Likert de cuatro puntos que reflejan el grado de acuerdo respecto a una serie de situaciones relacionadas con el contenido del trabajo. Las categorías de respuesta se distribuyen desde el "total desacuerdo" (1) hasta el "total acuerdo" (4). El instrumento está compuesto por 8 factores (empleo de habilidades, control sobre la tarea, demandas psicológicas, demandas físicas, apoyo social de compañeros, apoyo social del supervisor, apoyo social y autonomía) que hacen referencia a las demandas y recursos laborales presentes en cualquier tipo de trabajo. Es un instrumento que ha sido ampliamente utilizado en población trabajadora con discapacidad intelectual (Alcover y Pérez, 2010; Flores et al., 2011) y que cuenta con adecuadas propiedades psicométricas $(\alpha=0,73)$.

También se utilizó la Encuesta sobre indicadores de Calidad de Vida Laboral diseñada por Jenaro y Flores (2006) y utilizada en estudios previos con trabajadores con discapacidad intelectual (Flores, 2008; Jenaro et al., 2010). La encuesta contiene preguntas relacionadas con diversos factores de calidad de vida laboral tales como: valoración de estresores laborales (compuesto por 12 items a los que se responde en una escala tipo Likert de 10 puntos; $\alpha=$ 0,87), satisfacción laboral (formado por 5 items a los que se responden en una escala tipo Likert de 4 puntos; $\alpha=0,79$ ). Además, la Encuesta contiene una pregunta destinada a conocer la valoración subjetiva del trabajador sobre su calidad de vida laboral. En este caso se le pide al trabajador que puntúe su calidad de vida laboral en una escala continua de 10 puntos, donde 1 es la mínima puntuación y 10 es la máxima puntuación. Finalmente en la Encuesta se incluyen 
apartados referidos a datos sociodemográficos y otras variables relacionadas con características contextuales del trabajo.

Así mismo, se utilizaron las subescalas de Agotamiento (AG) y Cinismo (CY) del Cuestionario “Burnout” de Maslach-General Survey (MBI-GS, Schaufeli, Leiter, Maslach y Jackson, 1996) para evaluar el burnout. Se trata de una medida de autoinforme compuesta por 10 ítems destinados a evaluar el síndrome de quemarse por el trabajo en cualquier tipo de trabajo y que puede ser completado por cualquier tipo de trabajador. Dichos ítems se puntúan según una escala tipo Likert de 0 ("Nunca") a 6 ("Siempre") puntos que indica la frecuencia con que cada uno de los ítems es experimentado. El agotamiento (AG) hace referencia a sentimientos de fatiga, agotamiento y pérdida de energía experimentados por el trabajo en general. Por su parte, el cinismo o despersonalización (CY), refleja la actitud fría y distante del trabajador y su indiferencia respecto al contenido del trabajo y hacia el propio trabajo en sí mismo. Puntuaciones elevadas en ambas subescalas son indicativas de burnout. Existe acuerdo en considerar las dimensiones de agotamiento y cinismo como las dimensiones centrales del síndrome (Maslach, Schaufeli y Leiter, 2001). En el presente estudio se obtuvieron unos índices de fiabilidad con valores alfa de Cronbach de 0,78 para la subescala de agotamiento (AG) y de 0,72 para la subescala de cinismo (CY), lo que apoya su consistencia interna. En su aplicación a trabajadores con discapacidad intelectual se utilizó la adaptación realizada por Flores (2008).

De manera adicional, se incluyeron tres preguntas abiertas cuyo objetivo era recabar información sobre aspectos potenciadores de la satisfacción laboral. En este sentido las preguntas fueron:

1) ¿Te sientes preparada para desempeñar tu trabajo?; ¿En qué medida la formación que has recibido previamente te ha ayudado?;

2) ¿Qué apoyos recibes en el trabajo?; 3) ¿En qué medida tu empresa te ayuda a mejorar tu calidad de vida en el trabajo?.

Las respuestas fueron transcritas y analizadas según el modelo de análisis de contenido cualitativo propuesto por Mayring (2000). 


\subsection{Procedimiento}

Los datos se recogieron a lo largo del año 2012. Para ello se contó con el apoyo de una Fundación que gestiona diversos programas de Empleo con Apoyo (ECA) en la Comunidad de Madrid. Una vez confirmada la participación de dicha Fundación, miembros del equipo de investigación se desplazaron para llevar a cabo las entrevistas de evaluación con las trabajadoras con discapacidad interesadas en participar en el estudio. El criterio de inclusión fue que las participantes tuviesen edad laboral y se encontrasen en activo en el momento de realización del estudio. Así mismo, todas las participantes colaboraron de manera voluntaria y ofrecieron por escrito su consentimiento informado. En todos los casos se garantizó el anonimato y la confidencialidad de las respuestas emitidas.

\section{Resultados}

Para dar respuesta a los objetivos planteados se utilizaron estadísticos de contraste univariados (análisis de varianza) y estadísticos descriptivos, fundamentalmente. Los datos fueron analizados con el programa estadístico SPSS v. 15 (2006); el nivel de confianza establecido fue de $\alpha=0,05$. Los resultados de las preguntas abiertas fueron analizados con el procesador Atlas.ti 5, un software de análisis de datos cualitativos que facilita la localización y recuperación de datos, y el establecimiento de relaciones entre diferentes elementos.

En respuesta a nuestro primer objetivo, en la Tabla 1 se presentan los resultados sobre la percepción de las demandas laborales por parte de las trabajadoras. Se puede observar cómo, respecto a los estresores, los aspectos que más estrés suscitan son los relacionados con el hecho de tener que llevar a cabo gran cantidad de tareas $(M=4,89)$, así como la producción diaria $(\mathrm{M}=4,70)$ y el exceso de responsabilidad $(\mathrm{M}=4,27)$. Por su parte, la sobrecarga laboral (cuya puntuación máxima es de 4 puntos) también se percibe con una intensidad media $(\mathrm{M}=2,54)$, al igual que las demandas físicas del puesto de trabajo $(M=2,31)$. En términos generales puede decirse, sin embargo, que los estresores no son percibidos de modo muy intenso, dado que en ningún caso las puntuaciones superan el valor de 5 sobre 10 puntos. Respecto a los recursos, se puede observar cómo aquellos relacionados con el apoyo social son percibidos con mayor intensidad frente a los recursos relacionados con el contenido del trabajo. 
Desigualdad de género, inclusión laboral y riestos psicosociales: Evidencias en trabajadoras

Tabla 1. Estadísticos descriptivos de demandas laborales y recursos

\begin{tabular}{|l|c|c|c|c|c|}
\hline Estresores & $\mathbf{n}$ & Mínimo & Máximo & Media & $\begin{array}{c}\text { Desviación } \\
\text { Típica }\end{array}$ \\
\hline E. Horarios/turnos & 64 & 1 & 10 & 2,36 & 2,73 \\
\hline E. Cantidad Tareas & 64 & 1 & 10 & 4,89 & 3,39 \\
\hline E. Responsabilidad & 64 & 1 & 10 & 4,27 & 3,35 \\
\hline E. Tareas Repetitivas & 64 & 1 & 10 & 3,92 & 3,29 \\
\hline E. Poca Formación & 64 & 1 & 10 & 4,17 & 3,62 \\
\hline E. Producción diaria & 64 & 1 & 10 & 4,70 & 3,62 \\
\hline E. Poco Descanso & 64 & 1 & 10 & 2,77 & 2,73 \\
\hline E. Relación Jefe & 64 & 1 & 10 & 3,53 & 3,66 \\
\hline E. No feedback desempeño & 64 & 1 & 10 & 3,91 & 3,43 \\
\hline E. Relación compañeros & 64 & 1 & 10 & 3,52 & 3,46 \\
\hline E. No cambiar tareas & 64 & 1 & 10 & 2,48 & 2,57 \\
\hline E. Cambiar/Rotar & 64 & 1 & 10 & 2,42 & 2,91 \\
\hline Sobrecarga Laboral & 64 & 1 & 4 & 2,54 & 0,62 \\
\hline Demandas Físicas & 64 & 1 & 4 & 2,31 & 0,83 \\
\hline Empleo de habilidades & 64 & 1 & 4 & 2,81 & 0,51 \\
\hline Control sobre la tarea & 64 & 1 & 4 & 2,81 & 0,65 \\
\hline Autonomía & 64 & 1 & 4 & 2,81 & 0,46 \\
\hline Apoyo social supervisor & 64 & 1 & 4 & 3,15 & 0,68 \\
\hline Apoyo social compañeros & 64 & 1 & 4 & 3,14 & 0,60 \\
\hline
\end{tabular}

(Fuente: elaboración propia)

Por su parte, respecto al porcentaje de trabajadoras que manifiestan experimentar estrés en su lugar de trabajo, cabe destacar que un $47 \%$ contestó afirmativamente a la pregunta “Consideras que tu trabajo es estresante?” frente al 53\% que manifestó no experimentar estrés laboral. Respecto a la presencia de estrés laboral de tipo crónico o burnout, las puntuaciones obtenidas en cada una de las dimensiones del síndrome de burnout fueron recodificadas en los niveles alto, medio y bajo, atendiendo a los baremos propuestos por los autores originales del instrumento.

Así en la Figura 1 se observa cómo un 20,3\% de las trabajadoras con discapacidad intelectual presentan un elevado agotamiento y un 43,8 \% muestra un elevado cinismo o despersonalización. Lo que indica una presencia moderada de este tipo de estrés en la muestra de trabajadoras. 
Figura 1. Distribución de frecuencias de niveles de burnout (Fuente: elaboración propia)

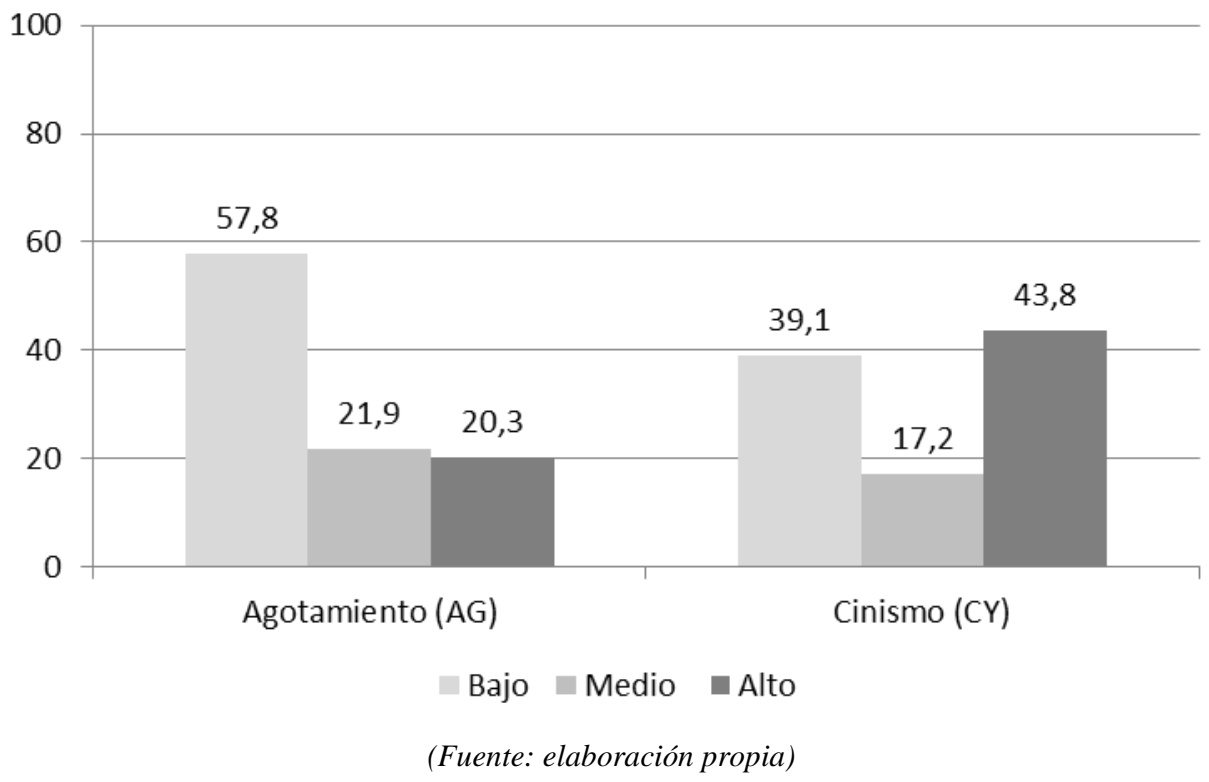

En cuanto a otras variables relacionadas con la salud laboral, preguntamos a las trabajadoras si en el último año habían solicitado consulta médica por problemas de estrés derivados del trabajo. Los resultados obtenidos pusieron de manifiesto que un $25 \%$ de las trabajadoras $(\mathrm{N}=16)$ habían solicitado consulta médica por este motivo frente a un $75 \%$ $(\mathrm{N}=48)$ que no solicitó dicha atención. Además, un $22 \%$ de las trabajadoras habían solicitado la baja laboral en el último año por problemas relacionados con el trabajo $(\mathrm{N}=14)$ frente al 78,1\% ( $\mathrm{N}=50)$ que contestó negativamente a esta cuestión. También se preguntó a las trabajadoras sobre la realización de conductas saludables (p.e. dormir horas suficientes, realizar actividad física, practicar deporte, destinar tiempo para el ocio) y de conductas insalubres (p.e fumar en exceso y consumir alcohol de manera abusiva).

En la Tabla 2 se aprecia la presencia de un mayor porcentaje de conductas saludables en la muestra de trabajadoras, si bien no son nada despreciables los porcentajes obtenidos en lo referido a la ausencia de practicar deportes y/o hacer ejercicio. 
Tabla 2. Porcentaje de conductas relacionadas con la salud

\begin{tabular}{|l|l|l|}
\hline Conductas Saludables/Insalubres & Sí (\%) & No (\%) \\
\hline Dormir horas suficientes & 87,5 & 12,5 \\
\hline Realizar actividad física & 51,6 & 48,4 \\
\hline Practicar deporte & 54,7 & 45,3 \\
\hline Destinar tiempo ocio & 73,4 & 26,6 \\
\hline Fumar en exceso & $3 \%$ & $97 \%$ \\
\hline Consumir alcohol de forma abusiva & $0 \%$ & $100 \%$ \\
\hline
\end{tabular}

(Fuente: elaboración propia)

En segundo lugar, y objeto de dar respuesta al objetivo consistente en identificar la presencia de diferencias significativas en la percepción de demandas laborales, recursos y satisfacción y calidad de vida laboral percibida, en función de si el trabajo es percibido o no como fuente de estrés, en la Tabla 3 se presentan los resultados obtenidos tras el análisis de varianza. Se puede observar la presencia de diferencias significativas en todas las demandas analizadas, exceptuando tan sólo los estresores relacionados con la cantidad de producción diaria, donde no se obtuvieron diferencias $\left(F_{(1,62)}=2,563\right.$, p>,05). Por tanto, las trabajadoras que manifiestan experimentar estrés en su lugar de trabajo también perciben con mayor intensidad las demandas laborales (p.e. cantidad de tareas, poco tiempo de descanso diario, escasez de formación, tareas repetitivas, sobrecarga laboral, demandas físicas) y perciben, por su parte, una menor satisfacción laboral. Respecto a los recursos, no se encontraron diferencias significativas en función del estrés percibido $(\mathrm{p}>0,05)$.

Tabla 3. Estadísticos Descriptivos y significación de las diferencias (Anova) entre las demandas laborales, recursos, satisfacción y calidad de vida laboral en función de la percepción de estrés laboral

\begin{tabular}{|l|c|c|c|c|c|}
\hline Demandas-recursos-satisfacción-CVL & Estrés & $\mathbf{n}$ & Media & $\begin{array}{c}\text { Desviación } \\
\text { Típica }\end{array}$ & F \\
\hline E. Turnos & & & & & $8,000^{* *}$ \\
\hline & Sí & 30 & 3,33 & 3,18 & \\
\hline E. Cantidad Tareas & No & 34 & 1,50 & 1,91 & \\
\hline & & & & & $14,841^{* *}$ \\
\hline & Sí & 30 & 6,47 & 2,86 & \\
\hline E. Exceso Responsabilidad & No & 34 & 3,50 & 3,25 & \\
\hline & & & & & $7,086^{* *}$ \\
\hline & Sí & 30 & 5,40 & 3,18 & \\
\hline E. Tareas Repetitivas & No & 34 & 3,26 & 3,22 & \\
\hline & & & & & $9,656^{* *}$ \\
\hline
\end{tabular}




\begin{tabular}{|c|c|c|c|c|c|}
\hline Demandas-recursos-satisfacción-CVL & Estrés & $\mathbf{n}$ & Media & $\begin{array}{c}\text { Desviación } \\
\text { Típica }\end{array}$ & $\mathbf{F}$ \\
\hline & No & 34 & 2,79 & 2,49 & \\
\hline \multirow[t]{3}{*}{ E. Escasez Formación } & & & & & $9,454 * *$ \\
\hline & Sí & 30 & 5,20 & 3,65 & \\
\hline & No & 34 & 2,79 & 2,49 & \\
\hline \multirow[t]{3}{*}{ E. Producción Diaria } & & & & & 2,563 (n.s) \\
\hline & Sí & 30 & 5,47 & 3,62 & \\
\hline & No & 34 & 4,03 & 3,54 & \\
\hline \multirow[t]{3}{*}{ E. Poco tpo. descanso } & & & & & 21,559 (n.s.) \\
\hline & Sí & 30 & 4,23 & 3,21 & \\
\hline & No & 34 & 1,47 & 1,23 & \\
\hline \multirow[t]{3}{*}{ E. Relación Jefe } & & & & & $13,514 * *$ \\
\hline & Sí & 30 & 5,17 & 3,97 & \\
\hline & No & 34 & 2,09 & 2,66 & \\
\hline \multirow[t]{3}{*}{ E. No información desempeño } & & & & & $13,346 * *$ \\
\hline & Sí & 30 & 5,43 & 3,66 & \\
\hline & No & 34 & 2,56 & 2,59 & \\
\hline \multirow[t]{3}{*}{ E. Relación compañeros } & & & & & $13,582 * *$ \\
\hline & Sí & 30 & 5,07 & 3,59 & \\
\hline & No & 34 & 2,15 & 2,73 & \\
\hline \multirow[t]{3}{*}{ Sobrecarga laboral } & & & & & $26,082 * *$ \\
\hline & Sí & 30 & 2,90 & 0,40 & \\
\hline & No & 34 & 2,23 & 0,61 & \\
\hline \multirow[t]{3}{*}{ Demandas Físicas } & & & & & $5,995 *$ \\
\hline & Sí & 30 & 2,57 & 0,84 & \\
\hline & No & 34 & 2,08 & 0,75 & \\
\hline \multirow[t]{3}{*}{ Empleo Habilidades } & & & & & 2,409 (n.s.) \\
\hline & Sí & 30 & 2,71 & 0,51 & \\
\hline & No & 34 & 2,90 & 0,49 & \\
\hline \multirow[t]{3}{*}{ Control sobre la tarea } & & & & & 0,560 (n.s.) \\
\hline & Sí & 30 & 2,88 & 0,59 & \\
\hline & No & 34 & 2,75 & 0,70 & \\
\hline \multirow[t]{3}{*}{ Autonomía } & & & & & 0,101 (n.s.) \\
\hline & Sí & 30 & 2,79 & 0,36 & \\
\hline & No & 34 & 2,83 & 0,52 & \\
\hline \multirow[t]{3}{*}{ Apoyo social supervisor } & & & & & 0,715 (n.s.) \\
\hline & Sí & 30 & 3,07 & 0,70 & \\
\hline & No & 34 & 3,22 & 0,65 & \\
\hline \multirow[t]{3}{*}{ Apoyo social compañeros } & & & & & 0,003 (n.s.) \\
\hline & Sí & 30 & 3,14 & 0,63 & \\
\hline & No & 34 & 3,15 & 0,58 & \\
\hline \multirow[t]{3}{*}{ Satisfacción Laboral } & & & & & $12,761 * *$ \\
\hline & Sí & 30 & 3,12 & 0,54 & \\
\hline & No & 34 & 3,52 & 0,35 & \\
\hline \multirow[t]{3}{*}{ Calidad de Vida Laboral } & & & & & 2,821 (n.s.) \\
\hline & Sí & 30 & 7,80 & 3,14 & \\
\hline & No & 34 & 8,88 & 1,93 & \\
\hline
\end{tabular}

n.s.= no significativo; *significativo con $\mathrm{p}<0,05 ; * *$ significativo con $\mathrm{p}<0,01$

(Fuente: elaboración propia) 
Seguidamente, se presentan los resultados derivados del análisis de varianza para dar respuesta a nuestro tercer objetivo consistente en analizar la existencia de diferencias significativas en los factores de riesgo psicosocial (demandas y recursos) en función de los niveles de agotamiento (AG) y cinismo (CY) presentados. Así, en las Tablas 4 y 5 se presentan los resultados en las variables donde se obtuvieron diferencias significativas $(\mathrm{p}<0,05)$.

Como se puede apreciar en la Tabla 4, existen diferencias significativas en la mayor parte de las demandas y los recursos en función de los niveles de agotamiento experimentados por las trabajadoras con discapacidad. Así mismo, respecto al agotamiento (AG), los análisis post-hoc (Scheffé) pusieron de manifiesto que las trabajadoras con elevado agotamiento experimentaban con mayor intensidad las demandas laborales y percibían también, en menor medida, los recursos.

Por su parte, no se obtuvieron diferencias significativas en las demandas relacionadas con las tareas repetitivas $\left(F_{(2,61)}=0,493, \mathrm{p}>, 05\right)$, la producción diaria $\left(F_{(2,61)}=1,567, \mathrm{p}>, 05\right)$, los períodos de descanso $\left(F_{(2,61)}=3,095, \mathrm{p}>, 05\right)$, la ausencia de información sobre el desempeño laboral $\left(F_{(2,61)}=0,998, \mathrm{p}>, 05\right)$, la mala relación con los compañeros de trabajo $\left(F_{(2,61)}=1,213, \mathrm{p}>, 05\right)$ y las demandas físicas $\left(F_{(2,61)}=1,485, \mathrm{p}>, 05\right)$. Respecto a los recursos, tan sólo el control sobre el contenido de la tarea $\left(F_{(2,61)}=0,098\right.$, p>,05) no arrojó diferencias estadísticamente significativas respecto al resto de recursos.

Tabla 4. Estadísticos Descriptivos y significación de las diferencias (Anova) entre los factores de riesgo psicosocial (demandas y recursos) en función de los niveles en agotamiento (AG)

\begin{tabular}{|l|c|c|c|c|c|}
\hline Demandas-recursos-satisfacción-CVL & $\begin{array}{c}\text { Agotamiento } \\
\text { AG }\end{array}$ & $\mathbf{n}$ & Media & $\begin{array}{c}\text { Desviación } \\
\text { Típica }\end{array}$ & F \\
\hline E. Turnos & & & & & $5,398^{* *}$ \\
\hline & Bajo & 37 & 1,57 & 2,06 & \\
\hline & Medio & 14 & 2,71 & 2,12 & \\
\hline & Alto & 13 & 4,23 & 3,94 & \\
\hline E. Cantidad Tareas & & & & & $10,194^{* *}$ \\
\hline & Bajo & 37 & 3,46 & 2,77 & \\
\hline & Medio & 14 & 6,57 & 3,29 & \\
\hline & Alto & 13 & 7,15 & 3,23 & \\
\hline & & & & & $3,560^{*}$ \\
\hline
\end{tabular}




\begin{tabular}{|c|c|c|c|c|c|}
\hline Demandas-recursos-satisfacción-CVL & $\begin{array}{c}\text { Agotamiento } \\
\text { AG }\end{array}$ & $\mathbf{n}$ & Media & $\begin{array}{c}\text { Desviación } \\
\text { Típica }\end{array}$ & $\mathbf{F}$ \\
\hline \multicolumn{6}{|l|}{ E. Exceso Responsabilidad } \\
\hline & Bajo & 37 & 3,65 & 3,37 & \\
\hline & Medio & 14 & 3,93 & 3,08 & \\
\hline & Alto & 13 & 6,38 & 3,42 & \\
\hline \multirow[t]{4}{*}{ E. Relación Jefe } & & & & & $4,099 *$ \\
\hline & Bajo & 37 & 2,84 & 3,33 & \\
\hline & Medio & 14 & 3,07 & 3,12 & \\
\hline & Alto & 13 & 6,00 & 4,24 & \\
\hline \multirow[t]{4}{*}{ Sobrecarga laboral } & & & & & $4,897 *$ \\
\hline & Bajo & 37 & 2,38 & 0,68 & \\
\hline & Medio & 14 & 2,95 & 0,40 & \\
\hline & Alto & 13 & 2,56 & 0,37 & \\
\hline \multirow[t]{4}{*}{ Empleo Habilidades } & & & & & $9,513 * *$ \\
\hline & Bajo & 37 & 2,94 & 0,45 & \\
\hline & Medio & 14 & 2,93 & 0,39 & \\
\hline & Alto & 13 & 2,32 & 0,51 & \\
\hline \multirow[t]{4}{*}{ Autonomía } & & & & & $3,264 *$ \\
\hline & Bajo & 37 & 2,89 & 0,45 & \\
\hline & Medio & 14 & 2,87 & 0,42 & \\
\hline & Alto & 13 & 2,53 & 0,41 & \\
\hline \multirow[t]{4}{*}{ Apoyo social supervisor } & & & & & $3,222^{*}$ \\
\hline & Bajo & 37 & 3,30 & 0,67 & \\
\hline & Medio & 14 & 3,10 & 0,66 & \\
\hline & Alto & 13 & 2,77 & 0,61 & \\
\hline \multirow[t]{4}{*}{ Apoyo social compañeros } & & & & & $4,465 * *$ \\
\hline & Bajo & 37 & 3,22 & 0,57 & \\
\hline & Medio & 14 & 3,33 & 0,38 & \\
\hline & Alto & 13 & 2,73 & 0,72 & \\
\hline
\end{tabular}

*significativo con $\mathrm{p}<0,05 ; * *$ significativo con $\mathrm{p}<0,01$

(Fuente: elaboración propia)

Así mismo, en la Tabla 5, y respecto a los niveles de cinismo o despersonalización que presentan las trabajadoras, se encontraron diferencias significativas $(\mathrm{p}<0,01)$ en la práctica totalidad de las demandas laborales analizadas. También en este caso, los análisis post-hoc (Scheffé) revelaron que las trabajadoras con niveles medios y elevados de cinismo diferían significativamente en cuanto a la percepción de las demandas del entorno laboral. Así mismo, y 
al igual que ocurrió con el agotamiento (AG), no se encontraron diferencias estadísticamente significativas en las demandas relacionadas con el no obtener información sobre el desempeño profesional $\left(F_{(2,61)}=0,998, \mathrm{p}>, 05\right)$, el estrés derivado de la mala relación con los compañeros de trabajo $\left(F_{(2,61)}=1,213, \mathrm{p}>, 05\right)$ y las demandas físicas $\left(F_{(2,61)}=1,485, \mathrm{p}>, 05\right)$. Por su parte, en los recursos tan sólo se encontraron diferencias significativas en aquellos referidos al control sobre la tarea y la autonomía. En estos casos, los análisis post-hoc (Scheffé) evidenciaron que las trabajadoras con niveles elevados de cinismo percibieron con menor intensidad estos recursos laborales. Por otro lado, no se obtuvieron diferencias significativas $(p>0,5)$ en los recursos relacionados con el empleo de habilidades $\left(F_{(2,61)}=0,170, \mathrm{p}>, 05\right)$, el apoyo social del supervisor $\left(F_{(2,61)}=0,548, \mathrm{p}>, 05\right)$ y el apoyo social de los compañeros de trabajo $\left(F_{(2,61)}=0,576, \mathrm{p}>, 05\right)$, respectivamente.

Tabla 5. Estadísticos Descriptivos y significación de las diferencias (Anova) entre los factores de riesgo psicosocial (demandas y recursos) en función de los niveles en cinismo (CY)

\begin{tabular}{|l|c|c|c|c|c|}
\hline Demandas-recursos-satisfacción-CVL & Cinismo (CY) & $\mathbf{n}$ & Media & $\begin{array}{c}\text { Desviación } \\
\text { Típica }\end{array}$ & F \\
\hline E. Turnos & & & & & $7,136^{* *}$ \\
\hline & Bajo & 25 & 1,04 & 0,200 & \\
\hline & Medio & 11 & 4,27 & 4,54 & \\
\hline E. Cantidad Tareas & Alto & 28 & 2,79 & 2,53 & \\
\hline & & & & & $4,176^{*}$ \\
\hline & Bajo & 25 & 3,44 & 2,67 & \\
\hline & Medio & 11 & 6,09 & 3,61 & \\
\hline E. Exceso Responsabilidad & Alto & 28 & 5,71 & 3,52 & \\
\hline & & & & & $12,659^{* *}$ \\
\hline & Bajo & 25 & 2,80 & 2,27 & \\
\hline & Medio & 11 & 8,00 & 2,28 & \\
\hline E. Tareas Repetitivas & Alto & 28 & 4,11 & 3,46 & \\
\hline & & & & & $5,093^{* *}$ \\
\hline & Bajo & 25 & 3,40 & 2,91 & \\
\hline & Medio & 11 & 6,64 & 3,50 & \\
\hline E. Escasez Formación & Alto & 28 & 3,32 & 3,10 & \\
\hline & & & & & $5,006^{* *}$ \\
\hline & Bajo & 25 & 2,56 & 2,27 & \\
\hline & Medio & 11 & 4,64 & 4,05 & \\
\hline
\end{tabular}




\begin{tabular}{|c|c|c|c|c|c|}
\hline Demandas-recursos-satisfacción-CVL & Cinismo (CY) & $\mathbf{n}$ & Media & $\begin{array}{l}\text { Desviación } \\
\text { Típica }\end{array}$ & $\mathbf{F}$ \\
\hline \multirow[t]{4}{*}{ E. Producción Diaria } & & & & & $17,298 * *$ \\
\hline & Bajo & 25 & 3,24 & 2,96 & \\
\hline & Medio & 11 & 9,36 & 0,92 & \\
\hline & Alto & 28 & 4,18 & 3,38 & \\
\hline \multirow[t]{4}{*}{ E. Poco tpo. descanso } & & & & & $3,668^{*}$ \\
\hline & Bajo & 25 & 1,76 & 1,98 & \\
\hline & Medio & 11 & 4,18 & 3,02 & \\
\hline & Alto & 28 & 3,11 & 2,94 & \\
\hline \multirow[t]{4}{*}{ E. Relación Jefe } & & & & & $6,256 * *$ \\
\hline & Bajo & 25 & 1,68 & 1,88 & \\
\hline & Medio & 11 & 4,27 & 4,54 & \\
\hline & Alto & 28 & 4,89 & 3,89 & \\
\hline \multirow[t]{4}{*}{ Sobrecarga laboral } & & & & & $8,675^{* *}$ \\
\hline & Bajo & 25 & 2,19 & 0,68 & \\
\hline & Medio & 11 & 2,92 & 0,34 & \\
\hline & Alto & 28 & 2,70 & 0,48 & \\
\hline \multirow[t]{4}{*}{ Control sobre la tarea } & & & & & $5,817 * *$ \\
\hline & Bajo & 25 & 2,77 & 0,56 & \\
\hline & Medio & 11 & 3,36 & 0,43 & \\
\hline & Alto & 28 & 2,63 & 0,69 & \\
\hline \multirow[t]{4}{*}{ Autonomía } & & & & & $3,606^{*}$ \\
\hline & Bajo & 25 & 2,80 & 0,35 & \\
\hline & Medio & 11 & 3,12 & 0,33 & \\
\hline & Alto & 28 & 2,70 & 0,53 & \\
\hline
\end{tabular}

*significativo con $\mathrm{p}<0,05 ; * *$ significativo con $\mathrm{p}<0,01$

(Fuente: elaboración propia)

Finalmente, con objeto de dar respuesta al último objetivo consistente en conocer otros factores potenciadores de la satisfacción laboral en las trabajadoras con discapacidad a partir de sus propias opiniones, se utilizaron tres preguntas abiertas. La valoración de las respuestas emitidas requirió su codificación posterior, empleando los factores utilizados en los diferentes instrumentos de evaluación cuantitativa. En este sentido y respecto a la pregunta ¿te sientes preparada para desempeñar tu trabajo? ¿en qué medida la formación que has recibido previamente te ha ayudado?, la mayor parte de las respuestas ofrecidas por las trabajadoras $(\mathrm{N}=50)$ coinciden en señalar que los Programas Formativos previos al empleo (i.e. Formación Profesional, Programas de Garantía Social) les han ayudado a obtener y mantener su trabajo. Esto se pone de manifiesto, por ejemplo, en el siguiente comentario realizado por una trabajadora de 28 años ("yo estuve en unos cursos de Garantía Social y lo que aprendí me sirvió para conseguir mi trabajo actual. De hecho, cuando realicé las prácticas allí no ningún 
problema porque estaba preparada para realizar el trabajo"). Sin embargo, un aspecto que las trabajadoras $(\mathrm{N}=30)$ perciben como punto débil es el relativo a la formación respecto a las tareas específicas que tienen que realizar en el propio puesto de trabajo [( "al principio me sentía perdida porque no sabes lo que te vas a esperar el primer día de trabajo, no sabes cómo reaccionar, no sabes lo que te van a decir sobre lo que tienes que hacer hasta que van pasando los días y ya todo va cambiando-trabajadora de 32 años-”)].

Respecto a la pregunta ¿qué apoyos recibes en el trabajo?, los resultados obtenidos tras el análisis de los fragmentos textuales pusieron de manifiesto que las fuentes principales de apoyo para las trabajadoras con discapacidad intelectual provenían de los supervisores $(\mathrm{N}=45)$ y compañeros de trabajo $(\mathrm{N}=54)$, fundamentalmente. Ello se refleja por ejemplo en el siguiente tipo de comentarios: [("yo, por ejemplo, tengo mucho apoyo de mi jefe porque también pertenece a la fundación y eso requiere más el apoyo de él y muchas veces dicen los compañeros "ya habla el padre de la segunda hija" porque me habla asi y en general los compañeros de trabajo me ayudan bastante"-trabajadora de 33 años-); ("yo recibo mucho apoyo de mis compañeras que me explican muchas cosas y por ejemplo no me dejan anulada, sino que me dicen que soy una más, porque que yo tenga minusvalía no quiere decir que yo solamente esté ahí para trabajar. Si hay reuniones por lo que sea de alguna cosa, pues a mí me apuntan y yo voy con ellos"-trabajadora de 27 años-); ("mis compañeros me tratan como a otra compañera más"-trabajadora de 31 años-)]. Por tanto, el apoyo social se percibe como el principal recurso potenciador de la satisfacción en estas trabajadoras.

El análisis de las respuestas emitidas ante la última pregunta, ¿en qué medida tu empresa te ayuda a mejorar tu calidad de vida en el trabajo?, puso de manifiesto que la mayor parte de las trabajadoras $(\mathrm{N}=37)$ demandaban una mayor formación en el puesto de trabajo [("que me den más formación en las cosas que me permiten hacer bien mi trabajo, como la atención al cliente"-trabajadora de 35 años-); ("que nos aporten todo lo que necesitamos para poder desempeñar bien el trabajo"-trabajadora de 28 años)]. Además, otro de los reclamos estuvo relacionado con la autonomía y el control sobre las tareas [(“un poquitín de libertad para poder desempeñar bien mis tareas y por el otro lado hacer bien mi trabajo"-trabajadora de 42 años-); ("que me den más trabajo y que me permitan organizar a mi el orden de las tareas"'-trabajadora de 31 años)]. Además, también se evidenciaron otros aspectos relacionados con las oportunidades de promoción y mejora laboral. Tal es el caso de los siguientes comentarios vertidos por algunas trabajadoras [("yo me siento bien haciendo bien el trabajo y 
que luego sabes que tienes un trienio, que te valoran, que eres una más de la empresa y que te suben como una más y cada vez me suben más por valoración del trabajo y me dan cheques como todo el mundo igual"-trabajadora de 31 años-); ("que nos den más oportunidades, por ejemplo no solamente a mí, sino a gente como nosotros para que puedan trabajar en empresas"'-trabajadora de 33 años-)]. Finalmente, se evidenciaron comentarios referidos a la mejora en las condiciones laborales, concretamente en lo relacionado a la remuneración, [( "que subieran más al salario porque estuve ocho horas trabajando y cobraba una miseria para ocho horas, pero bueno un poquito más de salario no estaría mal"-trabajadora de 29 años.); ( "que este sueldo sólo me vale para vivir con mis padres porque como tuviera que vivir sola o pagar un alquiler no podría"-trabajadora de 33 años-)].

\section{Conclusiones}

El presente trabajo ha permitido conocer la realidad a la que se enfrentan muchas mujeres con discapacidad intelectual en sus puestos de trabajo. En este sentido, los resultados obtenidos ponen de manifiesto que poseer un empleo es una condición necesaria pero no suficiente para garantizar la satisfacción laboral en este colectivo. Así, hemos podido constatar cómo las mujeres con discapacidad de este estudio perciben también factores de riesgo psicosocial en sus lugares de trabajo que les generan estrés y afectan negativamente a su satisfacción y salud laboral. De hecho un $47 \%$ de las trabajadoras percibe su trabajo como estresante. Estos resultados coinciden con otros realizados en el colectivo de personas con discapacidad intelectual (Alcover y Pérez, 2010; Flores et al., 2011; Paredes, Fernández-Cid y Ruíz, 2012).

Por otro lado, los resultados sugieren que esas situaciones mantenidas en el tiempo tienden a cronificarse dando lugar a procesos de estrés laboral de tipo crónico o burnout. En esta línea, llaman la atención los elevados niveles de agotamiento y cinismo encontrados. Las condiciones en las que se desarrollan la mayor parte de los trabajos de las personas con discapacidad (i.e. contratos de media jornada, escasa remuneración, no tener oportunidades de promoción, etc.), además de otros factores relacionados con el con propio contenido del trabajo (i.e. escasa formación en las tareas propias del puesto de trabajo, sobrecarga laboral, etc.) o la doble discriminación a la que se enfrenta la mujer con discapacidad en el trabajo, podrían 
contribuir en la explicación de estos resultados ya que, al igual que sucede en las personas sin discapacidad, la mujer es más vulnerable a experimentar riesgos psicosociales en su entorno de trabajo, tal y como se ha puesto de manifiesto también en diferentes estudios (Cifre, Salanova y Franco, 2011). Por tanto, la propia situación de desigualdad experimentada por las mujeres con discapacidad podría contribuir a explicar estos resultados. No obstante, será necesario realizar más estudios con muestras más amplias y con mujeres pertenecientes a diferentes colectivos laborales para poder confirmar dichos hallazgos.

De gran interés son también los comentarios vertidos por las trabajadoras sobre los aspectos potenciadores de la satisfacción laboral. En este sentido, el apoyo social, tanto el que procede del entorno más próximo al trabajador (compañeros de trabajo) como el ofrecido por los jefes o supervisores, se considera el principal elemento de apoyo para las trabajadoras. Estos resultados coinciden ampliamente con estudios previos realizados con este colectivo (Alcover y Pérez, 2010; Flores, 2008; Izuzquiza y de la Herrán, 2010; Jenaro et al., 2010) por lo que el apoyo percibido en el contexto de trabajo es el principal elemento potenciador de la satisfacción de estas trabajadoras. Así mismo, resultan de interés las opiniones referidas a la importancia de la formación recibida y el reclamo de una mejor formación en las tareas propias del puesto de trabajo. Numerosos estudios coinciden en señalar la situación de desventaja a la que se enfrentan las mujeres en relación a los aspectos formativos (CERMI, 2012; Muriel, 2011; Pallisera y Rius, 2007), por lo que no debemos obviar esta cuestión. Así, una adecuada formación en el puesto de trabajo podría amortiguar los efectos del estrés percibido. La opinión de las trabajadoras también nos permite constatar una vez más la situación de desigualdad respecto a los salarios y oportunidades de promoción y mejora laboral a la que se enfrenta el colectivo de personas con discapacidad y que ha sido también puesto de manifiesto en numerosos estudios sobre el tema (Conde, Portillo y Shum, 2003; Jenaro et al., 2010; Pallisera y Rius, 2007).

Así pues, los resultados de este trabajo nos han permitido corroborar la consideración de que los trabajadores con discapacidad intelectual en general y las mujeres en particular, no constituyen una excepción ante los riesgos laborales psicosociales. Es más, suponen un grupo de riesgo con los que se debe intervenir con objeto de prevenir los mismos. Por tanto, realizar intervenciones que tengan como objeto reducir las demandas y potenciar los recursos debe ser una tarea de obligado cumplimiento para todas aquellas empresas y organizaciones 
comprometidas con la calidad y con la mejora de la salud de sus trabajadores. Dichas intervenciones requerirán una modificación de aspectos individuales y organizacionales.

Finalmente, no queremos terminar sin señalar una serie de limitaciones del presente trabajo, como las relacionadas con la obtención de datos a partir de muestras incidentales que no permiten generalizar los resultados obtenidos más allá de la muestra empleada. Otra de las limitaciones alude a la naturaleza transversal de los datos. Por tanto, es preciso utilizar diseños longitudinales que permitan corroborar las relaciones causales a través del tiempo y que nos permitan ponen en marcha intervenciones y evaluar su eficacia en la reducción de los riesgos psicosociales. Pese a las limitaciones indicadas, el presente trabajo constituye un acercamiento a la realidad laboral de las mujeres con discapacidad intelectual, ámbito aún necesitado de esfuerzos investigadores en el camino hacia la inclusión laboral.

\section{BIBLIOGRAFÍA}

- Agencia Europea para la Seguridad y Salud en el Trabajo. FACTS 53 (2004): Garantizar la Seguridad y la Salud de los trabajadores con discapacidad. Bélgica: Agencia Europea para la Seguridad y Salud en el Trabajo.

- Agencia Europea para la Seguridad y Salud en el Trabajo. FACTS 87 (2010): Diversidad de los trabajadores y evaluación de riesgos: garantizar la cobertura para todos. Bélgica: Agencia Europea para la Seguridad y Salud en el Trabajo.

- Alcover, Carlos Ma y Pérez, Vanessa (2010): Aspectos psicosociales de la integración de personas con discapacidad en el Mercado ordinario de trabajo. Madrid: $\quad$ Fundación Adecco.

- Alomar, Elizabeth (2004): El treball dels joves amb retard mental en entorns normalizats: análisi d'una realitat de treball amb support. Tesis Doctoral, Universidad Ramón Llull: Barcelona.

- Bramston, Paul y Fogarty, Gerard (1995): "Measuring stress in the mildly intellectually handicapped: The factorial structure of the Subjective Stress Scale”. En: Research in Developmental Disabilities, nº 16, pp. 117-131. 
- Berg, Elith (1981): “Administración de centros y talleres para deficientes mentales en Dinamarca”. En: Bonagent, no 7, pp. 33-51.

- CERMI (2012): La transversalidad de género en las políticas públicas de discapacidad (volumen I). Madrid: CERMI.

- CERMI (2013): II Plan Integral de Acción de Mujeres con Discapacidad 2013-2016. Madrid: CERMI.

- Cifre, Eva, Salanova, Marisa y Franco, Joan (2011): "Riesgos psicosociales de hombres y mujeres en el trabajo: ¿una cuestión de diferencias?”. En: Gestión Práctica de Riesgos Laborales, $\mathrm{n}^{\mathrm{o}} 82$, pp. 28-36.

- Conde, Ángeles, Portillo, Inés y Shum, Grace (2003): "Discapacidad y empleo: una perspectiva de género". En: Alternativas: Cuadernos de trabajo social, n 11 , pp. 59-86.

- Eggleton, Ian; Robertson, Sue; Ryan Justine y Kober, Ralph (1999): “The Impact of Employment on the Quality of Life of People with Intellectual Disabilities". En: Journal of Vocational Rehabilitation, nº 13, pp. 95-107.

- Farris, Bryan y Stancliffe, Roger (2001): "The co-worker training model: Outcomes of an open employment pilot project”. En: Journal of Intellectual \& Developmental Disability, n²6, pp.145-161.

- Flores, Noelia (2008). Calidad de vida laboral en empleo protegido: evaluación de la salud y de los riesgos psicosociales. Madrid: Consejo Económico y Social (CES).

- Flores, Noelia et al., (2011): "Understanding Quality of Working Life of Workers with Intellectual Disabilities”. En: Journal of Applied Research in Intellectual Disabilities, $\mathrm{n}^{\circ} 24, \mathrm{pp}$. $133-141$.

- INE (2013): Informe del mercado de trabajo de las personas con discapacidad estatal. Datos 2012. Madrid: Servicio Público de Empleo Estatal.

- Izuzquiza, Dolores y de la Herrán, Agustín (2010): Discapacidad intelectual en la empresa. Las claves del éxito. Madrid: Pirámide.

- Jenaro, Cristina (2004): “Trabajo y Discapacidad". En: Joaquín de Elena (ed.). Desigualdad Social y Relaciones de Trabajo. Salamanca: Ediciones Universidad de Salamanca, pp. 129-142. 
- Jenaro, Cristina y Bagnato, Ma José (2006): “Aplicación de la escala de calidad de vida de Schalock y Keith (1993) a usuarios de centros ocupacionales de Uruguay, sus familias y supervisores”. En Miguel Ángel Verdugo (dir.): Cómo mejorar la calidad de vida de las personas con discapacidad. Instrumentos y estrategias de evaluación. Salamanca: Amarú, pp. 177-198.

- Jenaro, Cristina y Flores, Noelia (2006): "Calidad de Vida Laboral en Empleo Protegido”. En: Miguel Ángel Verdugo (dir.). Cómo mejorar la calidad de vida de las personas con discapacidad. Instrumentos y estrategias de evaluación. Salamanca: Amarú, pp. 199-214.

- Jenaro, Cristina et al. (2002): "Supported employment in the international context: An analysis of processes and outcomes”. En: Journal of Vocational Rehabilitation, n 17, pp. 5-21.

- Jenaro, Cristina et al. (2010): "Calidad de vida laboral en trabajadores en riesgo de exclusión social: modelo explicativo, evaluación y propuestas de mejora”. En: Miguel Ángel Verdugo et al. (coor.): Aplicación del paradigma de calidad de vida. Salamanca: Publicaciones del INICO, pp. 117-136.

- Karasek, Robert (1985): Job Content Questionnaire and User's Guide. Columbia University.

- Kelloway, Kevin y Day, Arla (2005): "Building Healthy Workplaces: What We Known So Far”. En: Canadian Journal of Behavioral Sciences, n³7, pp. 223- 235.

- Ley 31/1995, de 8 de noviembre de Prevención de Riesgos Laborales. BOE núm 269 de 10 de noviembre, p.1011.

- Levi, Lennart y Levi, Inger (2000): Guidance on Work-related Stress. Spice of Life, or Kiss of Death? Luxembourg: Office for Official Publications of the European Communities.

- Lunsky, Yona y Bramston, Paul (2006): “A preliminary study of perceived stress in adults with intellectual disabilities according to self-report and informant ratings". En: Journal of Intellectual \& Developmental Disabilities, $\mathrm{n}^{\mathrm{o}} 31$, pp.20-27.

- Mank, David, Cioffi, Andrea y Yovanoff, Paul (1999): "The impact of coworker involvement with supported employess on wage and integration outcomes”. En: Mental Retardation, $\mathrm{n}^{\mathrm{o}}$ 37,pp. 383-394. 
- Marcos, María del Rosario (2009): "El largo recorrido hacia el reconocimiento social y económico del trabajo de las mujeres". En: Cuestiones de género: de la igualdad y de la diferencia, $\mathrm{n}^{\circ}$ 4, pp. 1-14.

- Maslach, Christina, Schaufeli, Wilmar y Leiter, Michael (2001): “Job Burnout”. En: Annual Review of Psychology, n52, pp. 397-422.

- Mayring, Philipp (2000): "Qualitative Content Analysis". En: Forum Qualitative Social Research, $\mathrm{n}^{\mathrm{o}}$ 2, pp.1-10.

- Muriel, Silvia (2011) (dir.): Estudio cualitativo sobre la situación laboral de las mujeres con discapacidad y/o trastorno mental. País Vasco: EHLABE

- Nucci, Maria y Reiss, Steven (1987): "Mental Retardation and emotionals disorders: A test for increased vulnerability to stress". En: Journal of Intellectual \& Developmental Disabilities, nº 13, pp. 161-166.

- Nuño, Laura y Torres, Pepa (2012): "Las mujeres con discapacidad y el empleo". En: CERMI (Eeds.): La transversalidad de género en las políticas públicas de discapacidad (volumen I).Madrid: CERMI, pp. 341-350.

- ONU (2006): Convención sobre los derechos de las personas con discapacidad.

- Pallisera, María y Rius, María (2007): “¿Y después del trabajo, qué? Más allá de la integración laboral de las personas con discapacidad”. En: Revista de Educación, no 342, pp. 329-348.

- Paredes, Milagros, Fernández-Cid, Matilde y Ruíz, Ma Josefa (2012); "Prevención de riesgos laborales entre las personas con discapacidad intelectual en los centros especiales de empleo". En: Cuadernos de Trabajo Social, nº 25, pp. 249-260.

- Parent-Thirion, Agnes, Fernández, Enrique, Hurley, John. y Vermeylen, Greet (2007): Fourth European Working Conditions Survey. Dubiln, Ireland: European Foundation for the Improvement of Living and Working Conditions.

- Peiró, José María y Bravo, María Jesús (1999): “Factores Psicosociales en la prevención de riesgos laborales: oportunidades y retos para la Psicología del Trabajo y de las Organizaciones". En: Revista de Psicología del Trabajo y de las Organizaciones, nº15, pp. 137-146. 
- Real Decreto 870/2007, de 2 de julio, por el que se regula el programa de empleo con apoyo como medida de fomento de empleo de personas con discapacidad en el mercado ordinario de trabajo. BOE número 168 de 14 de julio de 2007, pp.30618-30622.

- Salanova, Marisa, Gracia, Francisco y Peiró, José María (1996): "Significado del trabajo y valores laborales”. En: José María Peiró y Francisco Prieto (dirs.): Tratado de Psicología del Trabajo.Volumen II: Aspectos Psicosociales del Trabajo. Madrid: Síntesis, pp: 35-62

- Salanova, Marisa, Peiró, José María y Prieto, Francisco (1993): "El significado del trabajo: una revisión de la literatura”. En: José María et al. (eds.): Los jóvenes ante el primer empleo: el significado del trabajo y su medida. Valencia: Nau Llibres, pp. 21-41.

- Sauter, Steven, Murphy, Lawrence y Hurrell, Joseph (1990). "Prevention of Work-Related Psychological Disorders. A National Strategy Proposed by the National Institute for Occupational Safety and Health (NIOSH)”. En: American Psychologist, $\mathrm{n}^{\circ}$ 45, pp. 1146-1158.

- Schaufeli, Wilmar, Leiter, Michael, Maslach, Christina y Jackson, Susan (1996): “The MBI-General Survey”. En: Christina Maslach et al. (ed.) Maslach Burnout Inventory. Third Edition. Palo Alto, CA: Consulting Psychologists Press, pp.19-26.

- Silva, Manuel Carlos (2008): “Desigualdades de género”. En: Cuestiones de género: de la igualdad y la diferencia, $\mathrm{n}^{\mathrm{o}} 3$, pp. 13-54.

- SPSS, Inc. (2006): SPSS base 15 for windows user's guide. Chicago, IL: SPSS Inc.

- Super, Donald (1980): “A Life-Span, Life-Space Approach to Career Development” En: Journal of Vocational Behavior, $\mathrm{n}^{\circ}$ 16, pp.282-298.

- Winer, John (2000): “Quality of life and the work environtment: the relationship between integration in the work environtment and quality of life as perceived by individuals with mental retardation". En: Dissertation Abstract International Section A: Humanities and Social Science, $\mathrm{n}^{\circ}$ 61, pp. 2043. 